\title{
The Sun Service KRIM - diagnostic complex of solar activity
}

\author{
Alexander Volvach $^{1 *}$, Olga Gopasyuk ${ }^{1}$, and Inna Yakubovskaya ${ }^{1}$ \\ ${ }^{1}$ Radio Astronomy and Geodynamics Department, Crimean Astrophysical Observatory RAS, \\ Katsively, Crimea
}

\begin{abstract}
The radio astronomical diagnostic complex of solar activity, created on the basis of the radio telescope RT-22 and three small radiotelescopes united in the Sun Service KRIM located in coordinates longitude $33^{\circ} 59^{\prime} 30^{\prime \prime}$ latitude $44^{\circ} 23^{\prime}$ 52", conducts simultaneous observations in the wavelength range from $8 \mathrm{~mm}$ to $1.2 \mathrm{~m}$ in the monitoring mode and alerts. The Sun Service KRIM registered a series of strong outbreaks in the Sun in September 2017, when it was at a minimum of its activity. The information obtained by radio telescopes correlates well with data from other terrestrial and satellite observatories such as RSTN and GOES. Correlation coefficients are calculated and scattering diagrams for X-ray class flares X9.3 and X2.2 are constructed. The information from the radio telescopes of the Sun Service KRIM allows them to be used for daily monitoring of solar activity, further processing of data obtained in the course of scientific research, short-term forecast of space weather and analysis of its infuence on the Earth.
\end{abstract}

\section{Introduction}

The sun is very dynamic and plays a key role in the entire solar system. On the surface of the Sun, there are constant physical processes associated with the release of energy. During solar flares a lot of energy is released and this occurs particularly in the sunspot area. The study of solar activity is of undoubted interest in connection with its influence on a number of geophysical phenomena and on the ecological situation on Earth. Solar flares are extremely powerful explosions in the solar atmosphere. They are found near sunspots, usually along the line of separation (neutral line) between regions with oppositely directed magnetic fields. Physically, the outbreak is a reaction of the solar atmosphere to a sudden rapid process of energy release, probably of magnetic origin. The response affects mainly the chromosphere and the crown [1]. The perturbation caused by a solar flare is accompanied by the formation of radio emission. Solar radio emission is studied in a wide range, beginning with millimeter waves and ending with waves of length 15-20 m. From the side, the long wavelength range is limited by the increase in the opacity of the ionosphere with increasing wavelength, while the reception of millimeter waves is difficult due to the absorption of radio waves by molecules of $\mathrm{O}_{2}$ and $\mathrm{H}_{2} \mathrm{O}$ in the Earth's atmosphere [2].

\footnotetext{
*Corresponding author: volvach@bk.ru
} 


\section{Experimental setup and methodology}

In the radio astronomy laboratory of the Crimean Astrophysical Observatory, the Sun Service KRIM operates on the basis of radio telescopes RT-22, RT-3, RT-2 and RT-M. Small radio telescopes are equipped with radiometers for six discrete wavelengths ranging from $3 \mathrm{~cm}$ to $1.09 \mathrm{~m}$. The solar radiometric complex RT-22 CrAO contains receivers for wavelengths of 8 and $13 \mathrm{~mm}$ and polarimeters for waves $2.0,2.3,2.8$ and $3.5 \mathrm{~cm}$. The data of daily radio monitoring with solar activity are stored in real time in digital form and are exposed to the world solar service that includes 14 ground-based observatories in cooperation with orbital observatories.

The data obtained in the radio telescope RT-M in the form of dynamic spectra provide valuable information for studying various events in the solar corona such as flares, coronal mass ejections. Such data are useful for determining the physical phenomena responsible for the acceleration of particles on the Sun [3].

\section{Observations of flare activity in September 2017}

As of September 2017, there was a 24 cycle of minimum solar activity. From 4 to 10 September, through the western half of the visible sun disk, the active region NOAA 12673 (according to NOAA (National Oceanographic and Atmospheric Administration)), was increasing its area and supporting very active beta-gamma-delta magnetic groups. According to SWPC (Space Weather Prediction Center), 54 flares of class C, 26 flares of class M, 4 flares of class X were recorded in this region (Fig. 1, Fig. 2).

The Sun service KRIM observed these outbursts using the radio telescopes at centimeter, decimeter and meter wave bands. On September 6, 2017, from 11:50 to 13:00 UT, the radio telescope RT-M of the Sun Service KRIM recorded a powerful radio burst at $275 \mathrm{MHz}$ and $327 \mathrm{MHz}$ (Fig. 1). Radio burst has reached the max at 12:02 UT.

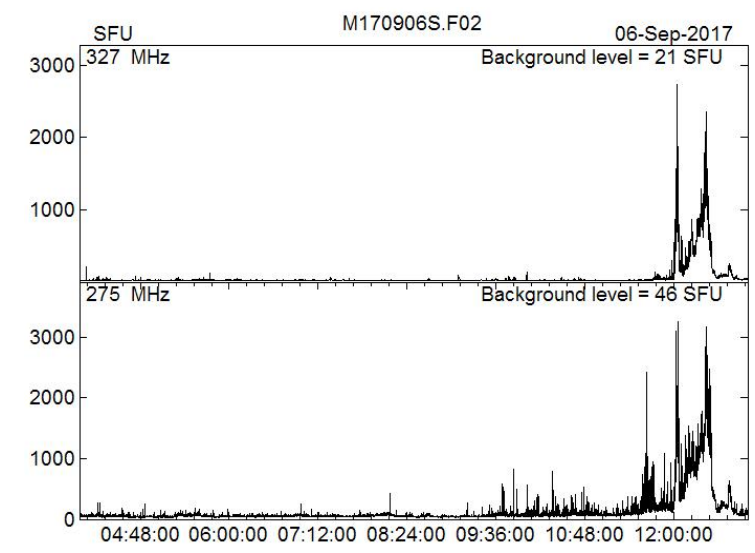

Fig. 1. Spectral fluxes of radio emission registered by RT-M. The chart clearly shows a strong radio burst that occurred at 12:02 UT with a power of $3260 \mathrm{SFU}\left(1 \mathrm{SFU}=10^{-22} \mathrm{Wt} / \mathrm{m}^{2} * \mathrm{~Hz}\right)$ at $275 \mathrm{MHz}$ and $2733 \mathrm{SFU}$ at $327 \mathrm{MHz}$.

At the same time, the satellites GOES 15 (Geostationary operational satellite for the study of the surrounding space) and GOES 14 also observed a powerful X-ray flash (the eighth largest in strength in the last 20 years). It was assigned X-ray class X9.3. This outbreak occurred in area of the Sun - NOAA 12673, located in heliographic coordinates S09 W34. In order to characterize the solar flare activity, in addition to radio emission, it is convenient to use the value of the X-ray flux, since during the flare its intensity can 
increase by several orders of magnitude. The NOAA also has a space weather scale for evaluating the effects of solar bursts on the radio communication on Earth and Earth's orbit. This flash is assigned class R3 - strong Radio Blackout occurred on 6 September from Region NOAA 12673 at 09:10 UT after X-ray class X2.2 burst. This leads to the disconnection of radio communication and the absence of RF communication, which mainly affects the frequency band from 3 to $30 \mathrm{MHz}$. There were also irregularities in the operation of navigation equipment operating at low frequencies, which lasted about an hour (https://www.swpc.noaa.gov).

A powerful flare was preceded by a weaker one with the X-ray class X2.2, it was also recorded in the centimeter wave band by the Sun's KRIM service with the RT-2 and RT-3 radio telescopes (Fig. 2, Fig. 3). This outbreak occurred in the same area of the Sun NOAA 12673, located in heliographic coordinates S08 W32.

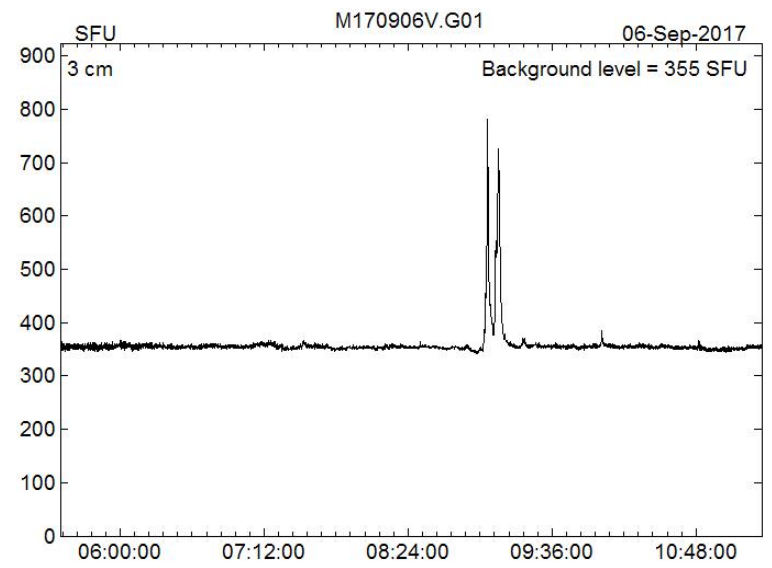

Fig. 2. Spectral flux of radio emission registered by RT-2 at a frequency of $10 \mathrm{GHz}$. The graph clearly shows that a strong radio burst occurred at 09:10 UT with a power of $691 \mathrm{SFU}$.

According to the SWPC burst of X-ray class X9.3 caused the formation of a type IV radio burst, followed by the formation of a type II radio wave burst propagating at a shock wave velocity of $1969 \mathrm{~km} / \mathrm{s}$, generated at the shock front (https://www.swpc. noaa.gov). Varieties of types of radio bursts can be determined by analyzing the data obtained by the radio telescope RT-M (Fig. 4). A powerful surge reached a maximum value at 12:02 UT and a series of bursts continued to be fixed by the equipment until 13:00 UT, when there was an automatic shutdown of the equipment. Observations in the field of radio waves at low frequency are the most informative tool for predicting the effects of solar flares. Spectral observations give a classification of bursts, based on well-defined features of radio emission, for example, at the velocity drift frequency, the width of the bands of individual burst details, and the structures of the harmonics. These burst characteristics are significant because they are related to the physical properties of the source.

\section{Conclusions}

Thus, the received and reliably confirmed information from the radio telescopes Sun service KRIM can be used for daily monitoring of solar activity. Daily data on the state of solar activity allow us to assess the radiation situation in the vicinity of the Earth, predict the stability of radio communications, the reliability of navigation systems, remote monitoring and control, and are the basis for studying physical conditions in the solar corona, which makes it possible to improve the accuracy of the forecast of solar activity. 


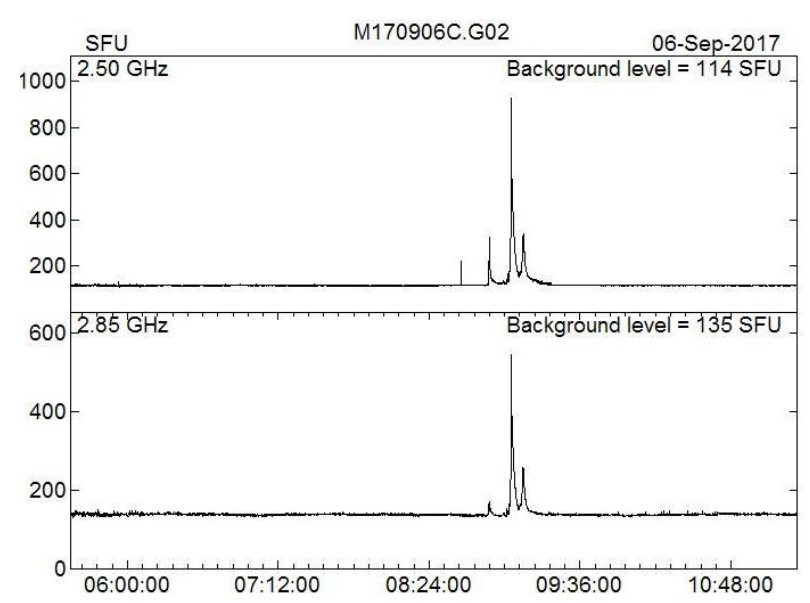

Fig. 3. Spectral fluxes of radio emission registered by RT-3 at a frequency of $2.5 \mathrm{GHz}$ and $2.85 \mathrm{GHz}$. The graph clearly shows that a strong radio burst occurred at 09:10 UT with a power of $691 \mathrm{SFU}$ at $2.5 \mathrm{GHz}$ and $578 \mathrm{SFU} 2.85 \mathrm{GHz}$.

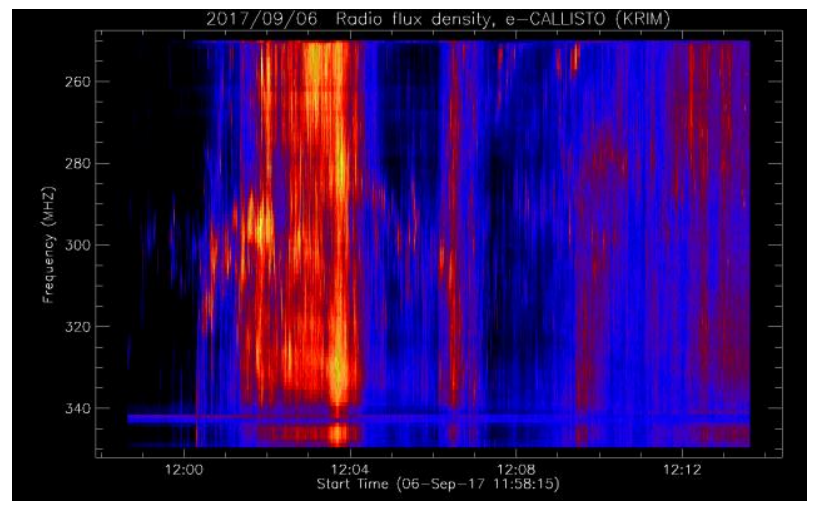

Fig. 4. Dynamic radio emission spectra X9.3 recorded by RT-M equipped with a spectrometer Callisto. Type IV radio bursts.

\section{References}

1. L.I. Miroshnichenko, The Physics of the Sun and the Sun-Earth Relationships, ed. by M. I. Panasyuk. Moscow: University Book Publishers, 174 pp. (2011)

2. V.V. Zheleznyakov, Advances in the physical sciences, p 114, LXIY, 1 (1958)

3. A.E. Volvach, Y.F. Yurovsky, News of the Crimean Astrophys. Obs., 112, 2, 179 (2016) 